\title{
Desk Rejecting: A Better Use of Your Time
}

\author{
Ben Ansell (Oxford University) \\ (benansell@gmail.com) \\ David Samuels (University of Minnesota) \\ dsamuels@umn.edu
}

February 2021 
Ben Ansell is Professor of Comparative Democratic Institutions in the Department of Politics and International Relations and Professorial Fellow, Nuffield College, Oxford University, and serves as co-editor of Comparative Political Studies. He can be reached at ben.ansell@ politics.ox.ac.uk.

David Samuels is Distinguished McKnight University Professor of Political Science at the University of Minnesota, and serves as co-editor of Comparative Political Studies. He can be reached atdsamuels@umn.edu.

Journal editing has become an increasingly challenging business in the past decade. When we took over as editors of Comparative Political Studies (CPS) in late 2013, the journal had accumulated a substantial backlog. Time between acceptance and publication had approached two years, partly because the number of submissions had tripled in a decade, reaching 599 that year. Submissions continue to increase on average about 7\% per year, and we received almost 850 papers in 2020. Even though we have doubled the number of articles CPS publishes annually in the last decade, we inevitably face a growing group of disappointed authors and exhausted reviewers.

To streamline the editorial process, we resolved to find ways to use authors' and reviewers' time more efficiently. A key decision was to increase the desk reject rate, which had hovered around $20 \%$ under the previous editor, to around $50 \%$. As part of this process, we make desk decisions "blindly" - that is, we decide whether to send a paper out for external review or not based on an anonymized manuscript. Increasing the desk reject rate has dramatically reduced the number of review requests we make, eliminated our backlog, and improved turnaround times. 
At the same time, conducting the internal review blindly hopefully prevents conscious and unconscious bias from affecting who gets reviewed (Brown and Samuels 2018).

Gibson (2020) anchors this symposium by arguing against desk rejecting. As authors, we understand what it feels like to receive a desk reject. Yet everyone has only 24 hours in a day, and as editors we know that our job is to focus on the journal's impact while using authors', reviewers' and our own time as efficiently as possible. In what follows we suggest that Gibson's critiques miss the mark and that his proposed alternatives to desk rejecting are either impractical or elitist. Subsequently, in the interests of editorial transparency, to expose the degree to which our editorial discretion achieves our goals of promoting the journal while using authors' and reviewers' time efficiently, we track the fate of every paper we accepted, desk rejected, and rejected after review during our first sixteen months as editors in 2013-14 (N=874), comparing publication rates, impact factors, and number of citations of papers we accepted, desk rejected, and rejected after review. Results actually suggest that we could increase our desk reject rate without sacrificing the quality of the journal's content - a decision that may prove inevitable as the number of submissions continues to rise.

\section{Responses to Gibson's Critique}

Gibson critiques editors" power in two ways. First, he suggests that "Desk rejecting violates the hallowed principle of peer-review" that colleagues should review journal submissions because editors cannot be experts on everything. This misunderstands the nature of academic journals. Academic journals publish peer-reviewed research - but are not in the business of providing peer review to anyone who submits. Gibson actually concedes this point, noting that some proportion of papers do not merit peer review. Of course, this raises the 
question of who should decide which papers should get desk rejected. Gibson implies that peer reviewers should make such choices, but this is illogical - it suggests editors should send papers out for review to decide which papers to send out for review. In any case it's the editors' job to make such decisions, based on criteria stated on their journal's submissions page which reflect editors' vision about a journal's content. Reviewers do not and cannot share the broad perspective that editors use to identify which papers might fit and which don't, since reviewers don't read the full slate of submissions a journal receives.

Second, Gibson suggests that "Desk rejecting is procedurally unfair," for two reasons. First, because editors' desk decisions take too long. That could be true, and editors should strive to make desk decisions quickly. But even if editors decided faster Gibson would still object based on the principle above. (At CPS, in 2019 papers spent an average of 19 days in internal review.) Second, because editors often provide no substantive justification for a desk reject, making it impossible for authors to improve their papers and making the decisions appear arbitrary. To the extent this is true it seems Gibson would still object even if editors offered substantive reasons for desk rejects - again because editors are not "peers."

Gibson's argument is also inconsistent. He agrees at one point that editors should not be mere bean counters, but then reverts to his argument that the power to desk reject goes too far. It is never clear where editors should reside on this continuum between "bean counters" and "arbitrary dictators." Instead, Gibson offers three suggestions to reduce desk rejection rates. However, all of these skirt around his core complaint about editorial power and instead seek to reduce submissions so that editors can rely on peer reviews for all decisions.

This focus implies that Gibson's real objection is not to an excess of editorial power but to an excess of weaker papers in the pipeline, which incentivizes editors to desk reject papers 
that might otherwise go out for review. Of course, it is not clear how an excess of weaker papers forces editors to desk reject stronger papers. Furthermore, reducing the number of submissions would not reduce editors' power at all.

In any case, Gibson's suggestions to reduce submissions are either impractical or elitist. First, Gibson suggests that journals could charge a submission fee and pay reviewers. He writes that some mechanism "might be established to subsidize those who claim they cannot afford the submission fee," but this is impractical. Who would manage the system, given the decentralized nature of journal ownership? And who would pay this subsidy? The answer cannot be "members of the association affiliated with that journal" because membership in an association is not and should not be a prerequisite for submission, and many journals (such as CPS) are not affiliated with an association. Gibson does recognize that many scholars might not be able to afford such fees. Productive scholars have multiple papers under review at any one time, often receive multiple rejections per year, and may have to resubmit the same papers multiple times. It is true that this model has been adopted by economics - but in many cases the fee is paid and the article still desk-rejected, which one presumes would be even less desirable for Gibson.

Gibson also suggests that journals could charge a publication fee in addition to a submission fee. Yet many or even most scholars could not afford such fees, particularly graduate students and scholars outside wealthy countries. Our discipline is increasingly global. Pressures to publish in top English-language journals are growing in every region, and scholars working outside wealthy countries often have fewer resources. On top of the growing demands for openaccess fees, more general publication fees are likely to prove very unpopular.

Finally, Gibson suggests that journals should discourage graduate students from submitting. Most graduate students, Gibson argues, do not "produce publishable work" and 
allowing "unworthy graduate student papers to muck up the review process is much too high a price to pay." This is simply elitist. One could equally suggest that most older scholars are long past their prime and should submit fewer papers. Both are equally offensive and counterproductive for the field. In any case the equilibrium outcome is easy to imagine: faculty would decide that their students' papers are worthy of submission but their colleagues' students' papers are not. Asking for lower graduate submissions has all the hallmarks of the collective action problems we study as political scientists - it is unlikely any individual graduate student would choose to refrain from submitting unless all graduate students were prevented from doing so.

In short, adopting Gibson's approach would impose monetary burdens on productive scholars and make the discipline more elitist, but would not resolve this deeper issue of "salami slice" or "minimum publishable unit" papers being submitted. Asking editors to increase the desk reject rate would not necessarily solve these problems either - but it would not be elitist, as long as editors do their internal reviews blindly. ${ }^{1}$

Gibson does actually identify the real challenge that editors face: the unceasing growth in submissions. Yet given this trend, like many editors we have chosen to judiciously conserve our most valuable resources - reviewers' time and good will - by not sending out for review papers that we regard as unsuitable. Desk rejecting is also a better use of authors' time than sending a paper out for review that has no chance. In some cases, it is both difficult to find reviewers and difficult to obtain promised reviews - months may pass that an author might not have wasted had he or she sent the paper to a more appropriate journal or spent more time working on the paper in the first place. Even with our desk-reject policy in place we have to ask five reviewers to get three reviews for the average manuscript, and it typically takes two weeks to secure them. A 
large number of papers require more than ten reviewer requests, delaying the process substantially.

\section{Results of An Internal Audit}

Are desk rejections actually an efficient use of editorial resources? All editors make both Type I and Type II errors - we accept some papers that will go unread and uncited and reject some papers that will go on to gain wide readership and a great number of citations. How frequent are such errors, for desk rejected papers as well as papers rejected after peer review?

Our job is to make judgment calls about which papers are already strong, and likely to be highly impactful if they are nudged in particular directions. How successful are we at making these judgment calls?

To answer these questions, we analyzed the fates of all submissions to CPS from the day we took over $(9 / 1 / 2013)$ through the end of $2014(\mathrm{~N}=874)$. This timeframe allows us to track what happens to papers we accepted, desk rejected, and rejected after review. As part of its standard business practice, CPS' publisher (SAGE Publications) tracks "lost citations" of submissions CPS rejected that other journals eventually published. However, SAGE can only track papers CPS rejects if the title remains unchanged when the paper subsequently appears in a different journal. Given this, SAGE could only track about 1/3 of the articles CPS rejected. We searched by hand for the remaining papers, using Google, Google Scholar, and authors' personal and/or professional web pages. ${ }^{2}$ In most cases the title was only slightly altered, and the paper rejected from CPS was easy to find. In cases where titles didn't obviously match, we compared the abstract of the CPS submission to the abstract of the eventually-published paper. Papers that 
subsequently appeared in a peer-review journal with the same author(s) and fundamentally similar arguments, variables and cases were regarded as "the same" as the CPS submission.

Once we compiled information on the fates of papers rejected from CPS, the question is how can we judge whether we had made a Type I or Type II error in rejecting a submission? It's not obvious what should count as a Type I error on our part, but "receiving zero citations" is a reasonable metric.

What should count as a Type II error, given the possibility that authors can revise after rejection? Answers to three questions provide a reasonable basis for such judgment. First, what proportion of rejected submissions were eventually published? Second, what proportion of rejected submissions were eventually published in higher-ranking journals? Of course, it is possible that an article that we reject that subsequently appears in a higher-ranking journal might never get cited, making it a Type I error for that journal and arguably not a Type II error for CPS. Thus, the third question is what proportion of submissions we rejected eventually receive a higher number of citations than the average paper that we accepted.

We used the two-year impact factor from the Journal Citation Reports to make crossjournal comparisons. This number is widely used to "rank" journals. To compare citations, we used the number of citations each article received in the year it was published (in a scheduled volume and issue, not online) plus two full calendar years, using the Social Sciences Citation Index. We would have preferred to count citations after four or five years, but $69 \%$ of all submissions to CPS in 2013-2014 that we rejected were published in 2016 or later. We wanted to compare the articles we accepted against as wide a sample of those we rejected that subsequently appeared elsewhere. Even with a two-year window for citations, we still have to exclude all 
papers CPS rejected that were published in 2018 or later, about $20 \%$ of the total (research was completed in September of 2020).

To be clear, only papers submitted to CPS in late 2013 through the end of 2014 are included in our analysis. Papers that we rejected that subsequently appeared elsewhere but only in 2018 or later are excluded entirely from the analysis, since they did not have the full two years to accumulate citations to make an "apples to apples" comparison with the set of papers (both accepted and rejected) that were in print for at least two years. Papers that were not published anywhere are given zero citations and are included in the analysis. It is certainly possible that some of these papers will eventually be published; the results below should be taken as a comparison of the "relative success of papers submitted to CPS in 2013-14" as of late 2020.

Table 1 provides results. First, we do make some Type I errors - a few of the papers we accepted received no citations after two years. However, this is true of fully $70 \%$ of the papers we desk rejected - and is even the case for more than half of the papers we rejected after review. This is partly because, as the second column reveals, a substantial proportion of papers we desk rejected and rejected after review did not appear in any peer-reviewed journal almost seven years after our editorial decision.

\section{Table 1 Here}

As for Type II errors, papers published in CPS receive on average almost five times the number of citations as papers we desk reject, and almost three times the number of citations as papers we rejected after review. Similar differences exist in terms of average impact factor. Again, these results are largely a function of the fact that so many rejected papers are never published. Still, we found that only $5.1 \%$ of papers we desk rejected and $11.1 \%$ of papers we rejected after review received more citations than the average paper we accepted. To emphasize 
the point: even after receiving full peer review, authors of only $11.1 \%$ of submissions we rejected subsequently revised their work so that the paper did as well or better as the average paper we accept - and only about one in twenty papers we desk rejected reached that mark. Likewise, only $2.7 \%$ of papers we desk rejected were eventually published in journals with a higher impact factor than CPS; the corresponding percentage for papers rejected after review is $6.2 \% .^{3}$

One might wonder if there are other systematic differences in the papers we desk reject compared to those we review and either accept or reject, not least the international origin of papers. However, breaking down submissions by country of origin of the corresponding author we see that, just limiting to submissions from the USA ( $46 \%$ of all submissions), accepted papers had 6.1 average citations and our CPS impact factor of 3.2, whereas review-rejected papers had 2.2 average citations and an average journal impact factor of 1.8, and desk-rejected papers had 0.95 citations and an impact factor of 1.3 .

Rejection should not be the end of a paper's life. The results of this audit suggest that sometimes we do make Type II errors - that we should not have rejected this or that paper. For desk rejected papers, however, this outcome is rare. And of course, the rejection may have been what spurred the authors to improve their paper. Overall, our decision process appears to be fairly efficient, undermining Gibson's claim that desk rejections are arbitrary and illegitimate. The fate of papers at CPS largely reflects their initial appropriateness and/or quality, given our mission to curate what the journal publishes. Overall, the vast majority of papers we desk reject either are not published after more than six years, appear in journals with a lower impact factor than CPS, and/or obtain fewer citations than the average paper published in CPS. We encourage editors of other journals to conduct similar audits as a way to gauge editorial effectiveness. 


\section{References}

Gibson, James. 2020. “Against Desk Rejects!” PS: Political Science and Politics.

Brown, Nadia and David Samuels. 2018. "Introduction to Gender in the Journals, Continued:

Evidence from Five Political Science Journals.” PS: Political Science \& Politics 51(4): 847-48.

Samuels, David. 2018. "Editorial Outcomes at Comparative Political Studies.” PS: Political Science and Politics 51(4) 854-858. 


\begin{tabular}{|c|c|c|c|c|}
\hline \multicolumn{5}{|c|}{ Table 1: CPS Internal Audit Results } \\
\hline Decision & $\begin{array}{c}\text { \% Not } \\
\text { Cited }\end{array}$ & $\begin{array}{c}\text { \% Not } \\
\text { Published }\end{array}$ & $\begin{array}{c}\text { Avg. } \\
\text { Citations }\end{array}$ & $\begin{array}{c}\text { Avg. } \\
\text { Impact } \\
\text { Factor }\end{array}$ \\
\hline Accepted & 8.6 & -- & 6.4 & 3.2 \\
\hline Rejected after Review & 56.1 & 35.7 & 2.3 & 1.9 \\
\hline Desk Rejected & 70.0 & 40.5 & 1.3 & 1.2 \\
\hline
\end{tabular}

${ }^{1}$ See Samuels (2018) for empirical evaluation of the extent to which CPS' triple-blind potentially process reduces gender bias.

${ }^{2}$ We thank Carly Coons and Yasmin Banishoraka for research assistance. Samuels conducted a second hands-on search for all papers that our RAs could not find anywhere online, using the author's name plus title words and keywords. If a paper could not be found after the second search, it was coded as "not published."

${ }^{3}$ In principle desk rejected papers might actually have a few months head start in finding a different publication outlet compared to papers rejected after review, but the higher citation rate for the papers rejected after review suggests differences in quality for desk-rejected versus peerrejected manuscripts. We thank Jim Garand for this suggestion. 